\title{
Aplicación de metodología DMAIC en el proceso productivo de fabricación de ladrillo artesanal
}

\section{Application of DMAIC methodology in the production process of artisan brick making}

\author{
NAVARRO-ENRÍQUEZ, Laura†*, TOVAR-VÁSQUEZ, Amado y RIVERA-MOJICA, Denisse
}

Universidad Tecnológica Paso del Norte, Procesos Industriales, México.

ID $1^{\mathrm{er}}$ Autor: Laura, Navarro-Enríquez / ORC ID: 0000-0001-6969-7529, CVU CONACYT ID: 775111

ID $2^{\text {do }}$ Coautor: Amado, Tovar-Vásquez / ORC ID: 0000-0002-7734-0631, CVU CONACYT ID: 1701809

ID $3^{\text {er }}$ Coautor: Denisse, Rivera-Mojica / ORC ID: 0000-0003-4382-2197, CVU CONACYT ID: 214879

DOI: $10.35429 / J T I P .2020 .10 .4 .1 .10$

Recibido: Enero 10, 2020; Aceptado: Junio 30, 2020

\section{Resumen}

La historia del ladrillo es la historia de la civilización, la arquitectura comenzó cuando se unieron 2 ladrillos. El ladrillo es un material de construcción más antiguo construido por el hombre. (W. P. Campbell,2004 y Sánchez, 2013). El presente trabajo tiene por objetivo identificar el proceso productivo de fabricación de ladrillo en la localidad de Ciudad Juárez por medio de la aplicación de la metodología DMAIC. El trabajo de investigación se basa en un muestreo exploratorio con visitas a las zonas de producción más importantes, específicamente en el km 20 en Ciudad Juárez Chih. observando el proceso de fabricación desde la Mezcla de las arcillas hasta la cocción de las unidades en el horno. Actualmente se ha encontrado que el molde de madera solamente permite obtener 3 ladrillos y pudiera mejorarse el molde para duplicar la producción, además pudiera adaptarse una mesa que le facilite al trabajador colocar el molde sin tener que inclinarse. Se ha encontrado que existe variabilidad en las dimensiones de los ladrillos entre una producción y otra, verificando las dimensiones de 1a quema del mes de enero. Analizando la producción de 500 ladrillos crudos en una jornada de 6 horas, tomando 5 productos cada hora.

Ladrillos, Procesos productivos, DMAIC, Variabilidad

\begin{abstract}
The history of the brick is the history of civilization, the architecture began when 2 bricks were joined. Brick is an older man-made building material. (W. P. Campbell, 2004 and Sánchez, 2013) he objective of this work is to identify the brick manufacturing production process in the city of Ciudad Juarez through the application of the DMAIC methodology. The research work is based on an exploratory sampling with visits to the most important production areas, specifically at $\mathrm{km} 20$ in Ciudad Juárez Chih. observing the manufacturing process from the mixing of the clays to the firing of the units in the oven. Currently it has been found that the wooden mold only allows to obtain 3 bricks and the mold could be improved to double the production, in addition a table could be adapted that makes it easier for the worker to place the mold without having to bend down. It has been found that there is variability in the dimensions of the bricks between one production and another, verifying the dimensions of the 1st burning in January. Analyzing the production of 500 raw bricks in a 6 -hour day, taking 5 products every hour.
\end{abstract}

Bricks, Productive process, DMAIC, Variability

Citación: NAVARRO-ENRÍQUEZ, Laura, TOVAR-VÁSQUEZ, Amado y RIVERA-MOJICA, Denisse. Aplicación de metodología DMAIC en el proceso productivo de fabricación de ladrillo artesanal. Revista de Tecnologías en Procesos Industriales. 2020. 4-10:1-10.

\footnotetext{
* Correspondencia al Autor: (Correo electrónico: laura.navarro@utpn.edu.mx)

$\dagger$ Investigador contribuido como primer autor.
} 


\section{Introducción}

La importancia de evaluar los parámetros que inciden en la productividad de una empresa determina un proceso de cambio que traerá beneficios significativos para la empresa, dichos parámetros están representados por factores externos como terrenos y edificios, materiales, energía, maquinaria y equipo y recurso humano. $\mathrm{O}$ bien factores internos como: disponibilidad de materia prima, mano de obra calificada, políticas estatales relativas a tributación y aranceles e infraestructura existente.

En las organizaciones se vive una intensa necesidad de desarrollo de calidad, de productividad y competitividad, pero no necesariamente a través de los procesos técnicos, sino de los factores humanos, que son el punto clave y estratégico de toda organización, ya que está comprobado que para que se promueva el conocimiento, se requiere de la disposición de las personas que lo poseen, el compartir dicho conocimiento y estar en la búsqueda de más, permite y garantiza un crecimiento multiplicativo en las organizaciones, y esto habla de personas que están abiertas al cambio, que tienen enfoques integrales, que observan el ritmo y del contexto global y detallan el contexto interno para adaptarlo a las necesidades de la organización.

A pesar de ser una empresa líder en el estado de Tabasco en todas las líneas que produce, y contar con la infraestructura y la tecnología básica para cubrir la demanda de la región, la permanencia de este sector por más de 15 años en el mercado no ha logrado un proceso de cambio significativo en su productividad, de aquí que se muestra un interés especial por conocer los factores que afectan la productividad de la empresa y con ello modificar su proceso interno (tecnológico, humano, material), cabe señalar que no existen antecedentes de haber realizado un estudio de esta línea de investigación en la empresa y por lo tanto se reconoce la importancia de los resultados que se obtengan de esta investigación. (Barranzuela, 2014)

\section{Descripción del método}

Para llevar a cabo el estudio de Aplicación De Metodología Dmaic en el proceso productivo de fabricación de ladrillo artesanal, se desarrolló la metodología siguiente:
Investigación de la información que provee el censo de población 2000 para la búsqueda de información del Directorio Estadístico Nacional de unidades económicas del INEGI(Denue/Since) para analizar el contexto socioeconómico de la producción de ladrillo, así como las normas aplicables respecto a este producto.

Para una mejor investigación y sustentación se han incluido visitas a las ladrilleras de nuestra zona en Ciudad Juárez, para identificar las posibles causas de las deficiencias que presentan en el producto final, sin dejar de lado las diferentes investigaciones sobre el tema que han realizado en otros países y en nuestro propio país y que han servido de mucha ayuda para complementar este trabajo citándolas en las referencias bibliográficas.

Identificar la variabilidad en las dimensiones (largo, ancho y alto) de los ladrillos entre una producción y otra, verificando las dimensiones de la quema al final del mes de Enero. Ya que se debe secar y dejar reposar el ladrillo en un lapso de 10 días. Analizando la producción de 500 ladrillos crudos en una jornada de 6 horas, tomando 5 productos cada hora.

Procesar y analizar la información a través tablas y graficas que permitan identificar el comportamiento de las variables estudiadas y también la aplicación de la metodología DMAIC y medición de los datos a través del control estadístico de calidad por medio de los gráficos de control para el monitoreo de las variables.

Determinar, de los factores encontrados, aquellos que inciden en las variables del contexto (variabilidad en dimensiones, falta de homogeneidad, producto con baja calidad, uniformidad y condiciones de trabajo anti ergonómico que afectan la eficiencia y eficacia del proceso de fabricación de Ladrillo, así como factores de riesgo por el proceso de trabajo artesanal) 
Interpretar el escenario de desarrollo que propicie el mejoramiento continuo del proceso productivo y la aplicación de la metodología DMAIC y la elaboración de una propuesta de mejora en el proceso actual de fabricación de ladrillos.

\section{Análisis a partir del método}

El estudio de la metodología DMAIC en el proceso de fabricación de ladrillos nos permite atacar proyecto de mejora o para solucionar problemas, siempre y cuando la magnitud de los mismos lo ameriten, o cuando no se conozca la causa raíz que está originando el efecto no deseado como son la insatisfacción del cliente y productos de baja calidad. Aplicando la metodología que incluye la aplicación de herramientas no estadísticas como son diagrama de flujo y de estadísticas básicas como son (gráficos de control, mapeo de proceso y control estadístico de calidad).

Esta metodología se divide en cinco etapas que se incluyen en el acrónimo DMAIC (Definir, medir, analizar, mejorar y controlar). (González, 2003).

\section{Definir el proyecto}

En esta etapa de definición se enfoca el proyecto, se delimita y se sienta las bases para su éxito. Por lo cual al finalizar se debe tener claro el objetivo del proyecto. (Pulido, 2008).

El problema a solucionar radica en la fabricación de ladrillos(tabiques) de $30 \mathrm{~cm}$ x 10 $\mathrm{cm}$ ancho $\mathrm{x} 5 \mathrm{~cm}$ altura medidas nominales según norma N-CMT-2-01-001-02 los cuales se ha encontrado que tienen una variabilidad en el proceso afectando la calidad del producto (Donderis, 2019).

El objetivo de este proyecto es identificar el proceso productivo de fabricación de ladrillo en la localidad de Ciudad Juárez por medio de la aplicación de la metodología DMAIC.

A través de la observación directa y la descripción de las etapas de producción de los ladrillos, se pudieron inferir posibles factores causantes de la mala calidad del producto (Donderis, 2019).
Se analizó el proceso productivo de la fabricación de ladrillo artesanal para lo cual se realizará una descripción del mismo y un diagrama de flujo. El alcance de este proyecto es tener ladrillos de buena calidad y disminuir la variabilidad en el proceso, así como mejorar el proceso actual y disminuir la fatiga al trabajador por medio de una mesa y el diseño de un nuevo molde con otro tipo de material más resistente, que dé una capacidad mayor de producción. Otra iniciativa es reducir los desperdicios generados por el proceso y eliminar actividades que no agreguen valor al producto. La descripción del proceso productivo es la siguiente:

\section{Etapa 1: Mezclado de las arcillas}

Se mezclan la arcilla negra aproximadamente medio metro y 1 metro de tierra blanca, se le agrega aserrín aproximadamente 2 carretillas y agua a consideración. Se bate con un azadón o pala hasta que toda la materia prima se incorpore, posteriormente se da una última batida con la mano.

\section{Etapa 2: Rebatido}

Cuando ya están mezcladas las arcillas se da una rebatida o boleado con la mano para que quede más fino el barro. Cuando ya no quedan grumos se considera que ya está lista para ponerse en los moldes.

\section{Etapa 3: Vaciar en moldes la arcilla y formar el ladrillo crudo}

Antes de colocar la mezcla se le pone arcilla sin batir para que no se pegue el ladrillo crudo. Se toma una porción con la mano y se coloca en el molde de madera que tiene 3 compartimentos, para lo cual se le quita los excedentes con una tablita para que quede más o menos parejo los ladrillos.

\section{Etapa 4: Tendido del ladrillo}

Una vez que se pone en el molde, se desmolda colocando en el piso para su secado. Pues el ladrillo es flexible y puede quebrarse fácilmente. Para este proceso debe durar 3 días tendido el tabique en el suelo para posteriormente apilarlo. El secado debe ser por un periodo de 10 días para poder meterse al horno a cocer. Para este estudio se analizó una producción de 500 ladrillos en una Jornada de 6 horas. 


\section{Etapa 5: Cocimiento en el Horno}

El horno está fabricado de paredes de adobe y destapado de arriba con un compartimento para poner los ladrillos y abajo se coloca la madera que consiste en paletas. Se introduce los ladrillos secos y se colocan en el horno. El horno debe estar prendido por 14 horas continuas con el ladrillo para su cocimiento.

\section{Etapa 6: Apilamiento ladrillo frio y cocido para su venta}

Posteriormente debe esperar 24 horas para que se enfrié el ladrillo para sacarlo y apilarlo y/o subirlo a la troca para su venta al cliente.

\section{Producción del ladrillo en México y los principales productores de ladrillos en México}

Según la información que brinda el INEGI, la producción de ladrillos tiene una influencia en todo el territorio nacional excepto en el estado de Campeche donde no se registra información de actividad de producción de ladrillos. El Censo Económico de 2020 reporta una caída en términos reales de $19 \%$ en el valor de la producción generado por las empresas. (INEGI, 2020). De las ocho regiones económicas en las que se integran todos los estados del país, la zona que tiene mayor participación en cuanto al número de unidades económicas es la zona oriente con 3,100 unidades (31\%) y está integrada por los estados de Hidalgo, Puebla, Tlaxcala y Veracruz, sin embargo, el estado con mayor relevancia dentro de esta región es Puebla. La segunda región es el occidente que representa el $26 \%$ de unidades económicas totales y está representado en orden de importancia por los estados de Jalisco, Michoacán, Nayarit y Colima. (SER pro, 2009).

\begin{tabular}{|l|r|r|r|}
\hline \multicolumn{1}{|c|}{ Estado } & \multicolumn{1}{c}{$\begin{array}{c}\text { Ladrilleras } \\
\text { registradas }\end{array}$} & \multicolumn{1}{c|}{$\begin{array}{c}\text { Encuestas } \\
\text { esperadas }\end{array}$} & $\begin{array}{c}\text { Encuestas } \\
\text { realizadas }\end{array}$ \\
\hline Hidalgo & 285 & 28 & 35 \\
\hline Tlaxcala & 29 & 3 & 3 \\
\hline Puebla & 4,500 & 440 & 4 \\
\hline Morelos & 24 & 2 & 0 \\
\hline $\begin{array}{l}\text { Ciudad de } \\
\text { México }\end{array}$ & 0 & 0 & 89 \\
\hline $\begin{array}{l}\text { Estado de } \\
\text { México }\end{array}$ & 803 & 78 & 202 \\
\hline Jalisco & 1994 & 195 & 249 \\
\hline Guanajuato & 2,366 & 231 & 2 \\
\hline Chihuahua & 2 & 2 & 1045 \\
\hline Total & 10,001 & 979 & \\
\hline
\end{tabular}

Tabla 1 Número de ladrilleras artesanales registradas por Estado

Fuente: INECC, 2016

\begin{tabular}{|c|c|c|}
\hline \multicolumn{2}{|c|}{$\begin{array}{l}\text { Temática: Población } \\
\text { orte: Municipio o delegación }\end{array}$} & \multirow[b]{2}{*}{ Porcentual } \\
\hline Indicador & Absoluto & \\
\hline ID & 676 & \\
\hline Clave geo estadística & 8,037 & \\
\hline $\begin{array}{l}\text { Población masculina } \\
\text { de } 50 \text { a } 59 \text { años }\end{array}$ & 42,506 & $6.4 \%$ \\
\hline \multicolumn{2}{|c|}{ Temática: Educación } & \\
\hline Indicador & Absoluto & Porcentual \\
\hline $\begin{array}{l}\text { Población de } 15 \text { años y } \\
\text { más sin escolaridad }\end{array}$ & 25,816 & $3 \%$ \\
\hline $\begin{array}{l}\text { Población masculina } \\
\text { de } 15 \text { años y más } \\
\text { sin escolaridad }\end{array}$ & 12,135 & $2.8 \%$ \\
\hline $\begin{array}{l}\text { Población de } 15 \text { años y } \\
\text { más con educación } \\
\text { básica incompleta }\end{array}$ & 308,340 & $35.5 \%$ \\
\hline
\end{tabular}

Tabla 2 Población y Educación de los trabajadores en las ladrilleras de Juárez, Chih.

Fuente: Scince, 2010

Con relación a la tabla 2 se puede decir que la mayor parte de la población en las ladrilleras y en específico las del kilómetro 20, la mayoría de los trabajadores tienen una edad promedio de 50 años y son los que realizan estas actividades de elaborar ladrillos y todos en $\mathrm{su}$ totalidad no cuentan con escolaridad.

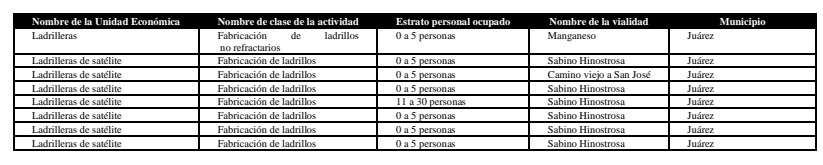

Tabla 3 Ladrilleras en Ciudad Juárez Chihuahua registradas

Fuente: (DENUE-INEGI, 2018)

\begin{tabular}{|l|l|}
\multicolumn{1}{|c|}{ Estado Combustible } \\
\hline Baja California & Leña limpia \\
\hline Coahuila & $\begin{array}{l}\text { Leña, madera y aceite } \\
\text { quemado }\end{array}$ \\
\hline Colima & Leña \\
\hline Chiapas & Cascaras de café \\
\hline Chihuahua & Leña(paletas) \\
\hline Durango & Leña y aserrín \\
\hline Sonora & Llantas, madera \\
\hline Querétaro & Basura, Poliéster \\
\hline
\end{tabular}

Tabla 4 Combustibles usados en la producción de ladrillos Fuente: Elaboración propia 


\begin{tabular}{|l|r|r|r|}
\hline \multicolumn{1}{|c}{$\begin{array}{c}\text { Medidas } \\
\text { nominales }\end{array}$} & $\begin{array}{r}\text { Fabricado } \\
\text { en } \\
\text { máquina }\end{array}$ & $\begin{array}{c}\text { Fabricado } \\
\text { a } \\
\text { mano }\end{array}$ \\
\hline Largo & 30 & 29 & 28 \\
\cline { 2 - 4 } & 20 & 19 & 18 \\
\hline Ancho & 10 & 9 & 13.5 \\
\cline { 2 - 4 } & 15 & 14 & 18.5 \\
\cline { 2 - 4 } & 20 & 19 & ----- \\
\cline { 2 - 4 } & 30 & 29 & ----- \\
\hline Altura & 5 & 4.5 & ----- \\
\cline { 2 - 4 } & 7.5 & 6.5 & 6 \\
\cline { 2 - 4 } & 10 & 9 & 8.5 \\
\cline { 2 - 4 } & 15 & 14 & ----- \\
\cline { 2 - 4 } & 20 & 19 & ----- \\
\cline { 2 - 4 } & 30 & 29 & ----- \\
\hline
\end{tabular}

Tabla 5 Dimensión de los Ladrillos y bloques cerámicos Fuente: Norma Mexicana NMX-C-404-ONNCE-2012 Fuente: Gobierno del Estado de México, 2004.

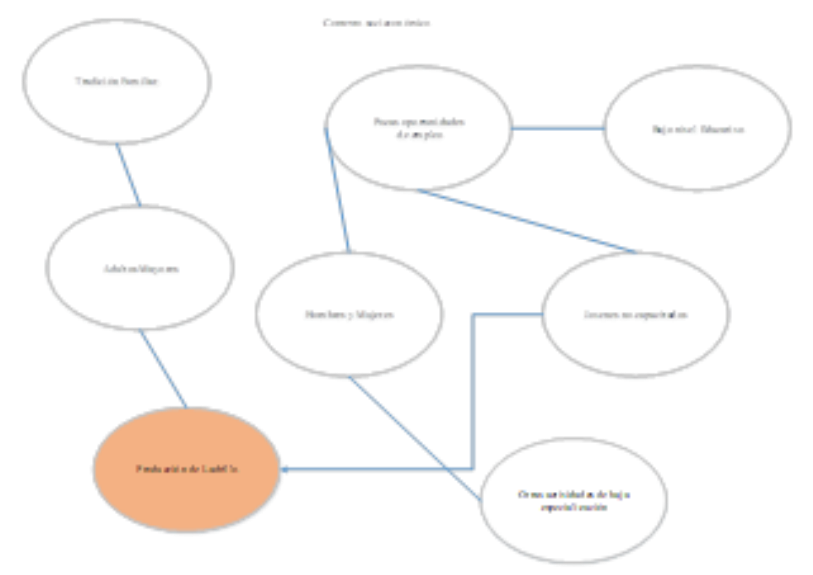

Figura 1 Contexto socioeconómico de la producción de ladrillo

Fuente: INEC, 2016

\section{Diagrama de flujo del proceso de fabricación de ladrillo}

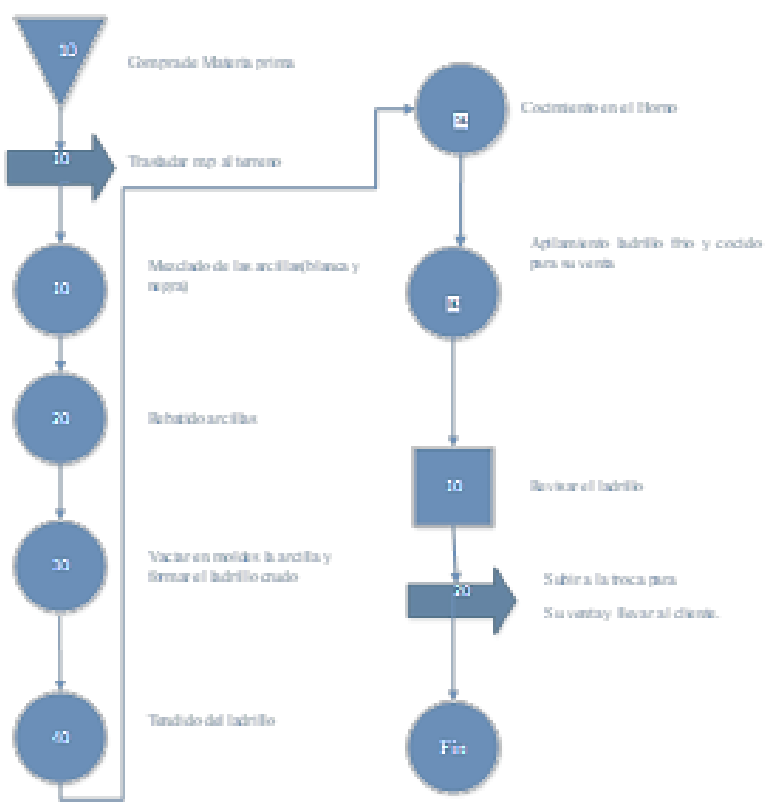

Figura 2 Diagrama de Flujo Proceso de fabricación del ladrillo

Fuente: Elaboración propia
Se puede definir al trabajo en el sentido ergonómico como la totalidad de la energía transformada por el hombre durante el cumplimiento de su tarea laboral. Ver Norma ISO 6385. Principios Ergonómicos de la concepción de sistemas de trabajo). (Melo, 2019)

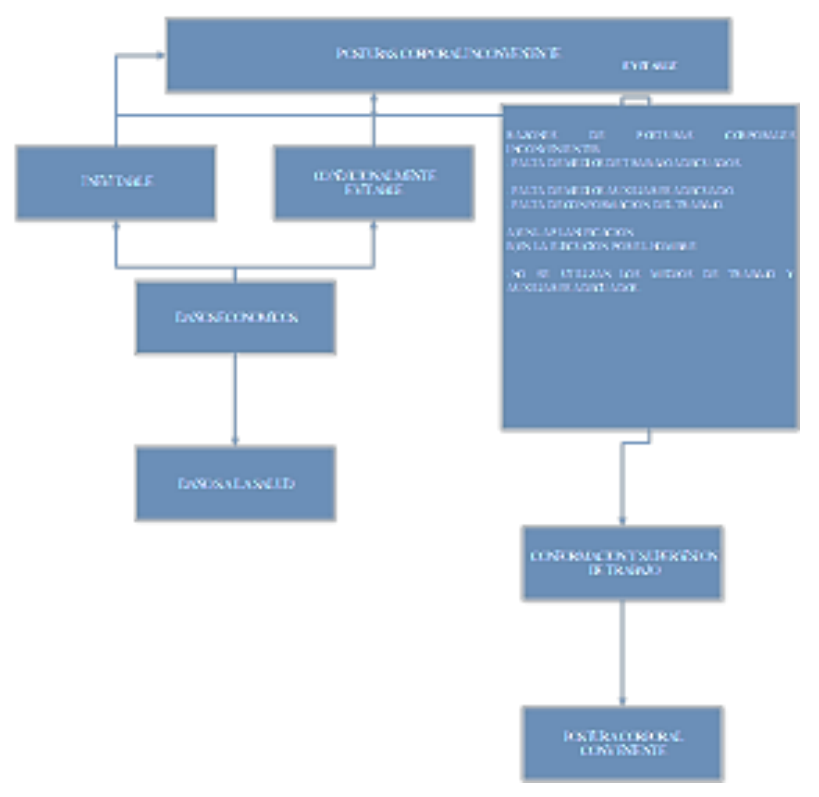

Figura 3 Posiciones corporales inconvenientes no evitables según (Melo, 2009)

\section{Medir}

Se realizó la recolección de los datos correspondientes a las variables de estudio que se muestran en la tabla 6.

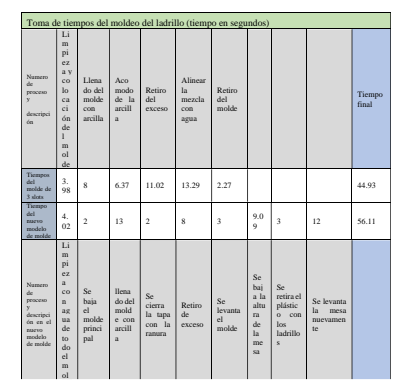

Tabla 6 Toma de tiempos del moldeo del ladrillo Fuente: Elaboración propia

Como las alturas de las mesas y las maquinas en general no son modificables verticalmente, sería necesario para ello adaptar la altura de trabajo a los hombres de elevada estatura, mientras que para las demás personas sería necesario utilizar tarimas o pedestales. La escala entre una mujer pequeña (5 percentil) y un hombre de gran estatura (95 percentil). 
Cada 10 días se hace una quema de ladrillos, por lo que se tomó 5 productos cada hora y se midieron de ancho, largo y alto en una Jornada de 6 horas. Y a continuación se visualiza la recolección de los datos en las tablas 8,9 y 10.

\begin{tabular}{|c|c|c|c|c|c|c|}
\hline \multirow{3}{*}{$\begin{array}{c}\text { Dimensiones en } \\
\mathrm{cm}\end{array}$} & \multicolumn{6}{|c|}{ Percentil } \\
\hline & \multicolumn{3}{|c|}{ Hombres } & \multicolumn{3}{|c|}{ Mujeres } \\
\hline & $5 \%$ & $\mathbf{5 0 \%}$ & $95 \%$ & $5 \%$ & $\mathbf{5 0 \%}$ & $95 \%$ \\
\hline $\begin{array}{l}\text { Alcance hacia } \\
\text { Adelante }\end{array}$ & 66.2 & 72.2 & 78.7 & 61.6 & 69 & 76.2 \\
\hline $\begin{array}{l}\text { Profundidad de } \\
\text { Caja }\end{array}$ & 23.3 & 27.6 & 31.8 & 23.8 & 29.5 & 35.7 \\
\hline $\begin{array}{l}\text { Alcance de los } \\
\text { Brazos } \\
\text { Hacia Arriba }\end{array}$ & 191 & 205.1 & 221 & 174.8 & 187 & 200 \\
\hline Altura del Cuerpo & 162.9 & 173.3 & 184.1 & 151 & 161.9 & 172.5 \\
\hline $\begin{array}{l}\text { Altura hasta el } \\
\text { Oido }\end{array}$ & 150.9 & 161.3 & 172.1 & 140.2 & 150.2 & 159.6 \\
\hline $\begin{array}{l}\text { Altura Hasta Los } \\
\text { Hombros }\end{array}$ & 134.9 & 144.5 & 154.2 & 123.4 & 133.9 & 143.6 \\
\hline $\begin{array}{l}\text { Altura hasta La } \\
\text { Cintura } \\
\text { (Parado) }\end{array}$ & 102.1 & 109.6 & 117.9 & 95.7 & 103 & 110 \\
\hline $\begin{array}{l}\text { Altura Hasta La } \\
\text { Mano } \\
\text { (Deje La Mano } \\
\text { Cerrada) }\end{array}$ & 72.8 & 76.7 & 82.8 & 66.4 & 73.8 & 80.3 \\
\hline $\begin{array}{l}\text { Ancho De La } \\
\text { Cadera( } \\
\text { Parado) }\end{array}$ & 31 & 34.4 & 35.8 & 31.4 & 35.8 & 40.5 \\
\hline $\begin{array}{l}\text { Ancho De } \\
\text { Hombros }\end{array}$ & 36.7 & 39.8 & 42.8 & 32.3 & 35.5 & 38.8 \\
\hline
\end{tabular}

Tabla 7 Medidas del hombre de pie Fuente: Norma DIN 3340

\begin{tabular}{|r|r|r|r|}
\hline \multicolumn{1}{|c|}{$\begin{array}{c}\text { Sub } \\
\text { grupo }\end{array}$} & \multicolumn{1}{c|}{ 1 } & \multicolumn{1}{c|}{$\mathbf{2}$} & \multicolumn{1}{c|}{$\mathbf{3}$} \\
\hline 1 & 6 & 5.5 & 5.6 \\
\hline 2 & 6.3 & 6.4 & 6 \\
\hline 3 & 6.8 & 6 & 6.2 \\
\hline 4 & 6 & 6 & 5.5 \\
\hline 5 & 5.5 & 5.4 & 5.5 \\
\hline 6 & 5.5 & 5.4 & 5.5 \\
\hline 7 & 5.4 & 5.5 & 5.6 \\
\hline 8 & 5.6 & 6 & 6.4 \\
\hline 9 & 6.5 & 6.4 & 6.5 \\
\hline 10 & 6.5 & 6 & 6 \\
\hline
\end{tabular}

Tabla 8 Observaciones del ancho de los Ladrillos Fuente: Elaboración propia

\begin{tabular}{|r|r|r|r|}
\hline \multicolumn{1}{|c|}{$\begin{array}{c}\text { Sub } \\
\text { grupo }\end{array}$} & \multicolumn{1}{c|}{ 1 } & \multicolumn{1}{c|}{ 2 } & \multicolumn{1}{c|}{$\mathbf{3}$} \\
\hline 1 & 18 & 20 & 18 \\
\hline 2 & 20 & 20 & 17.5 \\
\hline 3 & 18 & 19 & 18 \\
\hline 4 & 18 & 18 & 18.3 \\
\hline 5 & 18 & 18.1 & 18.2 \\
\hline 6 & 18 & 18.4 & 18 \\
\hline 7 & 18 & 17.5 & 20 \\
\hline 8 & 20 & 21 & 20 \\
\hline 9 & 21.5 & 22 & 19.5 \\
\hline 10 & 19.5 & 20 & 19.5 \\
\hline
\end{tabular}

Tabla 9 Observaciones de Largo de los Ladrillos Fuente: Elaboración propia

\begin{tabular}{|c|c|c|c|}
\hline \multirow{2}{*}{$\begin{array}{c}\text { Sub } \\
\text { grupo }\end{array}$} & \multicolumn{3}{|c|}{ Observaciones Ancho } \\
\hline & 1 & 2 & 3 \\
\hline 1 & 3.5 & 4 & 3.7 \\
\hline 2 & 3.8 & 3.6 & 3.9 \\
\hline 3 & 3.7 & 3.8 & 3.6 \\
\hline 4 & 3.5 & 3.5 & 3.5 \\
\hline 5 & 3.5 & 4 & 4 \\
\hline 6 & 4 & 4 & 4 \\
\hline 7 & 3.5 & 3.5 & 3 \\
\hline 8 & 3.2 & 3 & 3 \\
\hline 9 & 3 & 3.2 & 3 \\
\hline 10 & J & J & \\
\hline
\end{tabular}

Tabla 10 Observaciones de Alto de los Ladrillos Fuente: Elaboración propia

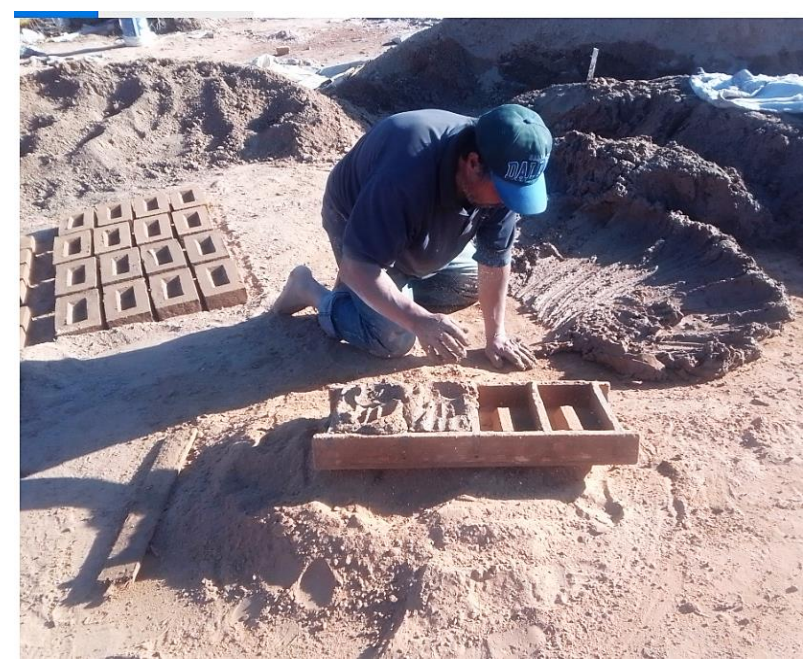

Figura 4 Vaciar arcilla en el molde Fuente: Elaboración propia

Como se puede observar en la Figura 4 el trabajador vacía la arcilla en el molde de madera, agachándose en cada momento que elabora el ladrillo o tabique.

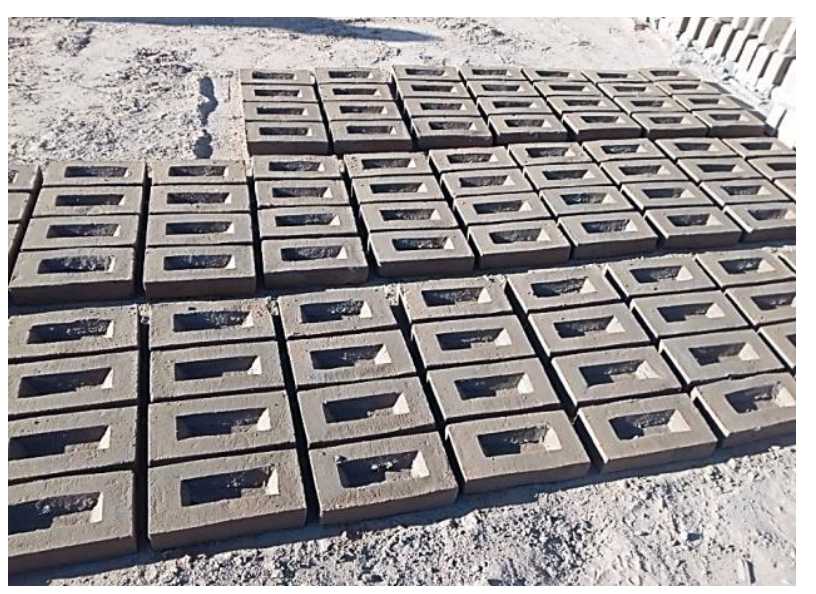

Figura 5 Tendido de ladrillo

Fuente: Elaboración propia

En la Figura 5 el trabajador desmolda el ladrillo y lo tiende en el piso. Lo deja secar al sol por al menos 2 días. 


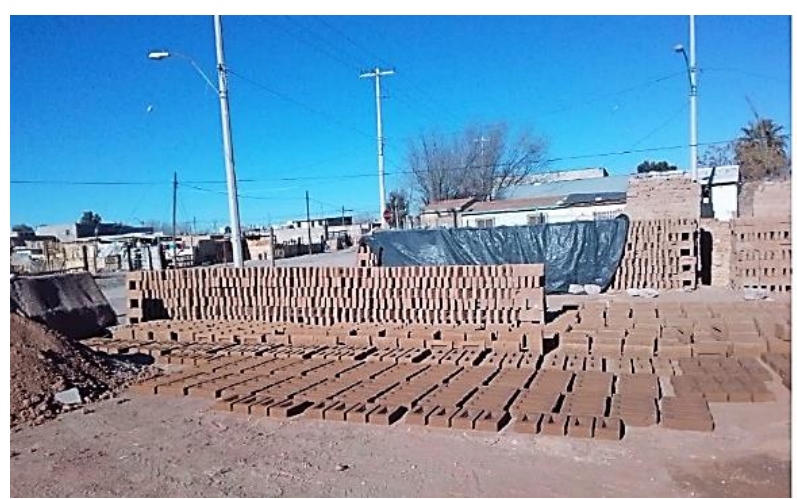

Figura 6 Apilar el ladrillo

Fuente: Elaboración propia

Una vez que el ladrillo está seco el trabajador apila el ladrillo como se muestra en la Figura 6 para su distribución y venta al cliente.

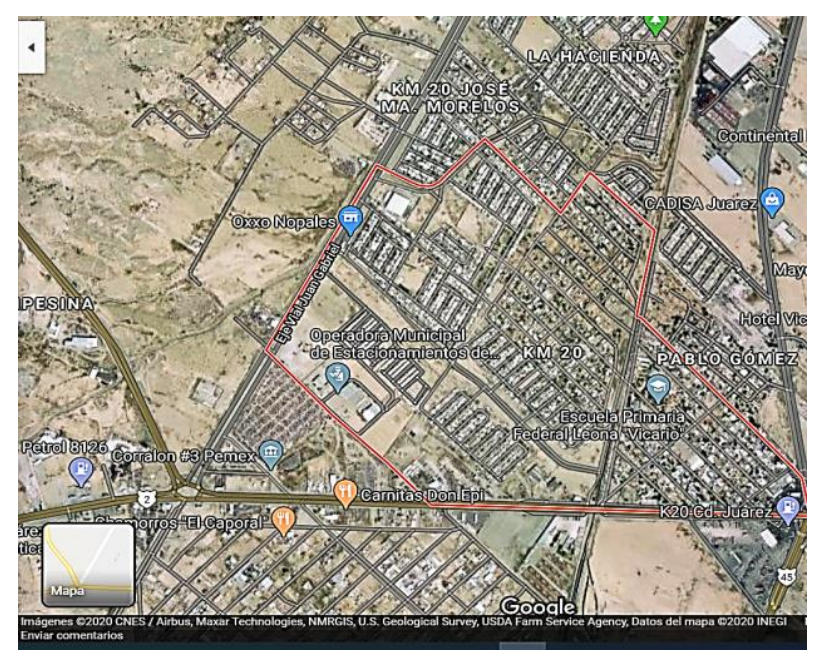

Figura 7 Zona de la Ladrillera km. 20 en Ciudad Juárez, Chih.

Fuente: Google Earth, 2020

\section{Resultados}

A continuación, se presentarán solamente aquellos resultados que observaron una variación significativa y que por lo tanto inciden en la productividad de la fabricación de ladrillo. En las siguientes gráficas se muestran el promedio de los limites inferior, superior, media y rango. Por medio de estos resultados de la información se da cuenta de que en las mediciones hay mucha variación utilizando el molde actual de 3 slots, por lo que se buscará solucionar este problema. Se puede observar que no todos los puntos están dentro de los límites estadísticos, tanto en el ancho, largo y alto. Por lo cual no se considera un sistema de producción estable, es probable que exista una causa especial que pudiera estudiarse a profundidad por medio de un análisis estadístico más detallado. Ver gráfico 2
Junio 2020 Vol.4 No.10 1-10

Como se puede observar en la tabla 6 de tiempos, el nuevo modelo de molde sobrepasa por 11.18 segundos, pero produciendo el doble de ladrillos que el molde original y eliminando el desgaste físico que implicaba agacharse en el molde original (Gutiérrez, 2009).

\begin{tabular}{|c|c|c|c|c|c|c|c|}
\hline \multicolumn{4}{|c|}{ Datos de medias } & \multicolumn{4}{|c|}{ Datos de Rangos } \\
\hline$\underline{\mathbf{X}}$ & LC X & LCS & LCI & $\mathbf{R}$ & LC $\mathrm{R}$ & LCS & LCI \\
\hline 5.700 & 5.917 & 6.326 & 5.507 & 0.5 & 0.4 & 1.0294 & 0 \\
\hline 6.233 & 5.917 & 6.326 & 5.507 & 0.4 & 0.4 & 1.0294 & 0 \\
\hline 6.333 & 5.917 & 6.326 & 5.507 & 0.8 & 0.4 & 1.0294 & 0 \\
\hline 5.833 & 5.917 & 6.326 & 5.507 & 0.5 & 0.4 & 1.0294 & 0 \\
\hline 5.467 & 5.917 & 6.326 & 5.507 & 0.1 & 0.4 & 1.0294 & 0 \\
\hline 5.467 & 5.917 & 6.326 & 5.507 & 0.1 & 0.4 & 1.0294 & 0 \\
\hline 5.500 & 5.917 & 6.326 & 5.507 & 0.2 & 0.4 & 1.0294 & 0 \\
\hline 6.000 & 5.917 & 6.326 & 5.507 & 0.8 & 0.4 & 1.0294 & 0 \\
\hline 6.467 & 5.917 & 6.326 & 5.507 & 0.1 & 0.4 & 1.0294 & 0 \\
\hline 6.167 & 5.917 & 6.326 & 5.507 & 0.5 & 0.4 & 1.0294 & 0 \\
\hline
\end{tabular}

Tabla 11 Datos de medias y rangos del ancho de ladrillo Fuente: Elaboración propia (2020)

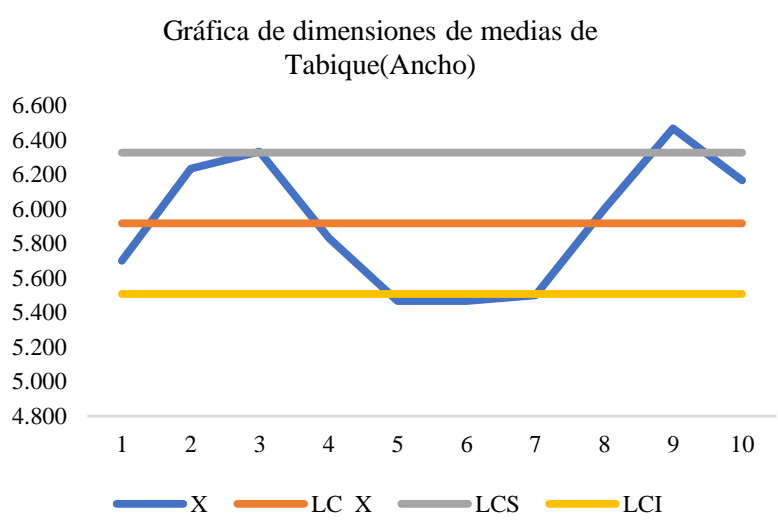

Gráfico 1 Observaciones de medias Tabique (Ancho) Fuente: Elaboración propia (2020)

Gráfica de dimensiones de rangos de Tabique(R Ancho)

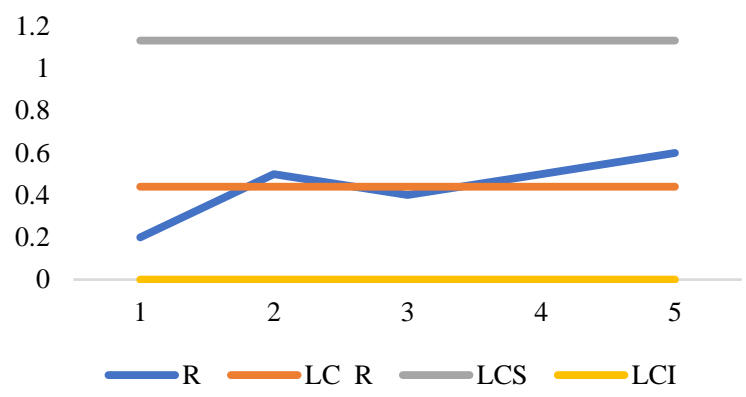

Gráfico 2 Observaciones de rangos de Tabique (Ancho) Fuente: Elaboración propia (2020) 


\begin{tabular}{|c|c|c|c|c|c|c|c|}
\hline \multicolumn{4}{|c|}{$\begin{array}{l}\text { Datos de medias } \\
\text { LC }\end{array}$} & \multicolumn{4}{|c|}{$\begin{array}{l}\text { Datos de Rangos } \\
\text { LC }\end{array}$} \\
\hline$\underline{x}$ & $\underline{x}$ & LCS & LCI & $\mathbf{R}$ & $\underline{\mathbf{R}}$ & LCS & LCI \\
\hline 18.667 & 19 & 20.320 & 17.680 & 2 & 1.29 & 3.319815 & 0 \\
\hline 19.167 & 19 & 20.320 & 17.680 & 2.5 & 1.29 & 3.319815 & 0 \\
\hline 18.333 & 19 & 20.320 & 17.680 & 1 & 1.29 & 3.319815 & 0 \\
\hline 18.100 & 19 & 20.320 & 17.680 & 0.3 & 1.29 & 3.319815 & 0 \\
\hline 18.100 & 19 & 20.320 & 17.680 & 0.2 & 1.29 & 3.319815 & 0 \\
\hline 18.133 & 19 & 20.320 & 17.680 & 0.4 & 1.29 & 3.319815 & 0 \\
\hline 18.500 & 19 & 20.320 & 17.680 & 2.5 & 1.29 & 3.319815 & 0 \\
\hline 20.333 & 19 & 20.320 & 17.680 & 1 & 1.29 & 3.319815 & 0 \\
\hline 21.000 & 19 & 20.320 & 17.680 & 2.5 & 1.29 & 3.319815 & 0 \\
\hline 19.667 & 19 & 20.320 & 17.680 & 0.5 & 1.29 & 3.319815 & 0 \\
\hline
\end{tabular}

Tabla 12 Datos de medias y rangos del largo de ladrillo Fuente: Elaboración propia (2020)

Gráfica de dimensiones de Tabique(Xbarra Largo)

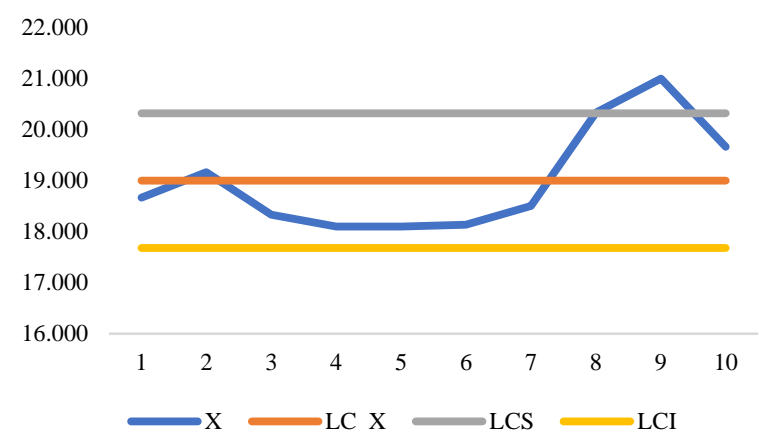

Gráfico 3 Dimensiones de medias Tabique (Largo) Fuente: Elaboración propia, (2020)

Gráfica de dimensiones de Tabique(R Largo)

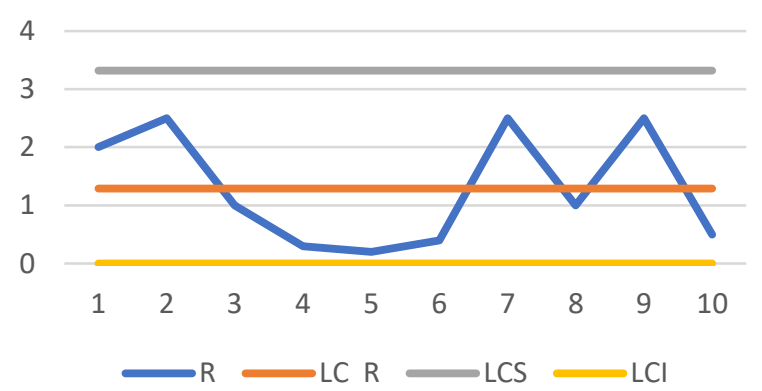

Gráfico 4 Observaciones de rangos de Tabique (Largo) Fuente: Elaboración propia, (2020)

\begin{tabular}{|c|c|c|c|c|c|c|c|}
\hline \multicolumn{4}{|c|}{$\begin{array}{l}\text { Datos de medias } \\
\text { LC }\end{array}$} & \multicolumn{4}{|c|}{ Datos de Rango } \\
\hline$\underline{x}$ & $\underline{\mathbf{X}}$ & LCS & LCI & $\mathbf{R}$ & $\underline{\mathbf{R}}$ & LCS & LCI \\
\hline 3.733 & 3.500 & 3.746 & 3.254 & \begin{tabular}{|l}
0.5 \\
\end{tabular} & 0.24 & 0.61764 & 0 \\
\hline 3.767 & 3.500 & 3.746 & 3.254 & 0.3 & 0.24 & 0.61764 & 0 \\
\hline 3.700 & 3.500 & 3.746 & 3.254 & 0.2 & 0.24 & 0.61764 & 0 \\
\hline 3.500 & 3.500 & 3.746 & 3.254 & 0 & 0.24 & 0.61764 & 0 \\
\hline 3.833 & 3.500 & 3.746 & 3.254 & 0.5 & 0.24 & 0.61764 & 0 \\
\hline 4.000 & 3.500 & 3.746 & 3.254 & 0 & 0.24 & 0.61764 & 0 \\
\hline 3.333 & 3.500 & 3.746 & 3.254 & 0.5 & 0.24 & 0.61764 & 0 \\
\hline 3.067 & 3.500 & 3.746 & 3.254 & 0.2 & 0.24 & 0.61764 & 0 \\
\hline 3.067 & 3.500 & 3.746 & 3.254 & 0.2 & \begin{tabular}{|l|l|} 
\\
\end{tabular} & 0.61764 & 0 \\
\hline 3.000 & 3.500 & 3.746 & 3.254 & 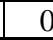 & 0.24 & 0.61764 & 0 \\
\hline
\end{tabular}

Tabla 13 Datos de medias y rangos del alto de ladrillo Fuente: Elaboración propia, (2020)
Gráfica de dimensiones de Tabique(Xbarra Alto)

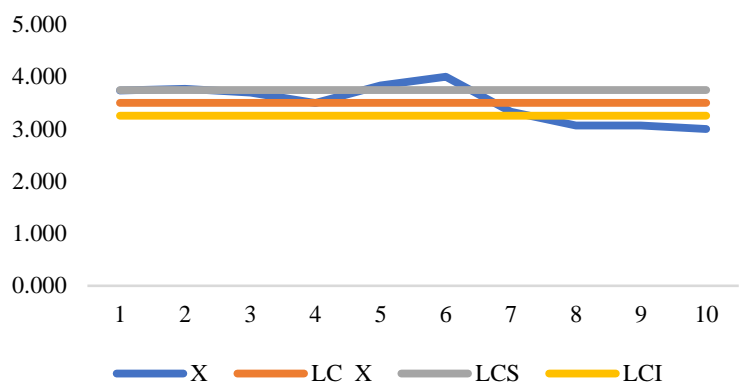

Gráfico 5 Dimensiones de medias Tabique (Alto) Fuente: Elaboración propia, (2020)

Gráfica de dimensiones de Tabique $(\mathbf{R}$ Alto)

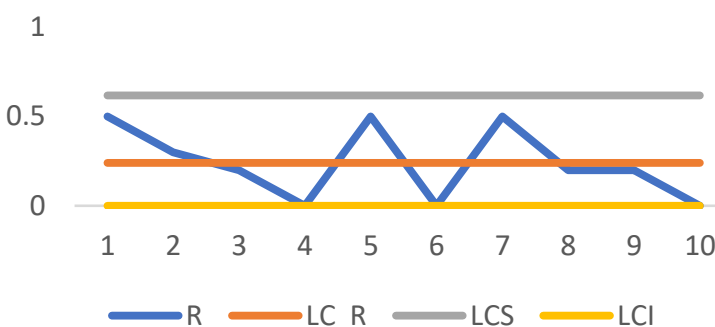

Gráfico 6 Observaciones de rangos de Tabique (Alto) Fuente: Elaboración propia, (2020)

\section{Agradecimiento}

Agradezco a la Universidad Tecnológica Paso del Norte por la oportunidad de publicar este artículo. A mi directora de Carrera Ángeles Holtzeimer Álvarez, Ing. Tovar Vásquez Amado, Ing. Rivera Mojica Denisse, Ing. Miriam Ramos, Ing. Luis Fernando Garza, Hernández Palomino Rene Jesús, Hernández Velázquez Erika Fabiola, por el apoyo brindando para la elaboración de este artículo.

\section{Propuesta}

\begin{tabular}{|c|c|c|c|c|c|c|}
\hline \multicolumn{7}{|c|}{ Cotización y datos del material } \\
\hline $\begin{array}{l}\text { Material } \\
\end{array}$ & \begin{tabular}{|l|} 
Empresa \\
\end{tabular} & Descripción & $\begin{array}{l}\text { Medidas } \\
\end{array}$ & Cantidad & $\begin{array}{l}\text { Datos } \\
\text { adicionales }\end{array}$ & Costos \\
\hline Llantas & Hágalo & Rotatorias & 3 pulgadas & 2 & & 450.00 \\
\hline Tubo & Hágalo & Fierro & $610 \mathrm{~cm} \mathrm{x}$ & 1 & & 450.00 \\
\hline Tornillos & Home Depot & Fierro & $\begin{array}{l}2^{2 " 1 / 4} \\
\text { pulgada }\end{array}$ & 50 & $\begin{array}{l}\text { Buscarrosca } \\
\text { negro }\end{array}$ & 54.50 \\
\hline Bisagra & Home Depot & Fierro & $3 " \times 3 "$ & 4 & & 342.00 \\
\hline Madera & Home Depot & Madera & $\begin{array}{l}4^{4 " x} 45 " x \\
40 "\end{array}$ & 4 & Personalizada & 1680.00 \\
\hline Barra & \begin{tabular}{|l|} 
Home Depot \\
\end{tabular} & Fierro & $\begin{array}{l}4 \mathrm{cmx} x \\
200 \mathrm{~cm} \mathrm{x} \\
4 \mathrm{~cm}\end{array}$ & 2 & Personalizada & 294.00 \\
\hline $\begin{array}{l}\text { Construcción y } \\
\text { corte }\end{array}$ & $\begin{array}{l}\text { Carpintería } \\
\text { Hernández }\end{array}$ & Madera & & & $\begin{array}{l}\text { Se cortaran las } \\
\text { piezzas para darle } \\
\text { forma al molde }\end{array}$ & 600.00 \\
\hline $\begin{array}{l}\text { Corte y } \\
\text { soldado de } \\
\text { metal }\end{array}$ & $\begin{array}{l}\text { Carpintería } \\
\text { Hernández }\end{array}$ & Fierro & & & $\begin{array}{l}\text { Se cortaran las } \\
\text { piezas para darle } \\
\text { forma al molde }\end{array}$ & 300.00 \\
\hline $\begin{array}{l}\text { Costo del } \\
\text { material }\end{array}$ & & & & & & 3270.50 \\
\hline $\begin{array}{l}\text { Costo de mano } \\
\text { de obra }\end{array}$ & & & & & & 900.00 \\
\hline $\begin{array}{l}\text { Costo de } \\
\text { producción }\end{array}$ & & & & & & 4170.50 \\
\hline
\end{tabular}

Tabla 14 Cotización del material para fabricar propuesta de molde

Fuente: Elaboración propia

NAVARRO-ENRÍQUEZ, Laura, TOVAR-VÁSQUEZ, Amado y RIVERA-MOJICA, Denisse. Aplicación de metodología DMAIC en el proceso productivo de fabricación de ladrillo artesanal. Revista de Tecnologías en Procesos Industriales. 2020 
Los principios de diseño de trabajo es adaptar la estación ergonómicamente al trabajador humano, por lo regular el diseño del trabajo se olvida cuando se busca el aumento de la productividad. Es necesario que el ingeniero industrial aplique los principios de diseño del trabajo para brindarle al operador una estación de trabajo libre de riesgo y minimizarle la fatiga por movimientos inadecuados. (Lopez, 2015).

La Figura 6 muestra el diseño del molde a 6 cavidades con una mesa ergonómica que se puede ajustar al tamaño del trabajador y cuenta con ruedas para su mejor manejo.

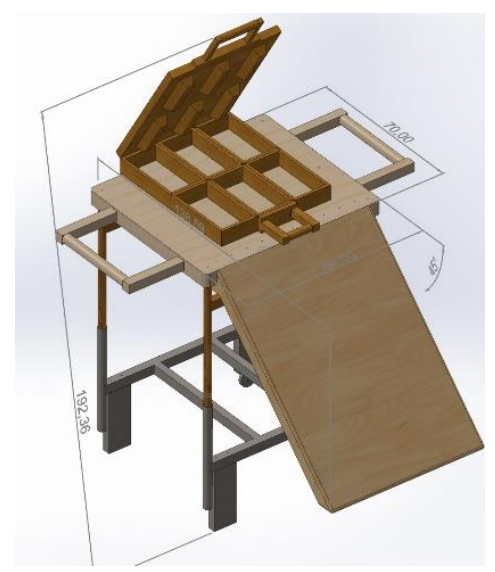

Figura 8 Propuesta del molde con mesa para aumentar la productividad

Fuente: Elaboración propia

Cabe mencionar que el costo de material es $\$ 3,270.50$ pesos, el costo de material será de $\$ 900.00$ pesos y el costo de producción de $\$ 4,170.00$ pesos.

El funcionamiento del molde consiste en facilitar al trabajador el moldeo de los ladrillos, reduciendo el esfuerzo y duplicando el moldeo de ladrillos pues, el molde que se utilizaba era de 3 slots y aparte el trabajador tenía que estar agachado para poder colocar el material y esto pudiera haber causado un daño en la columna a cierto plazo.

\section{Ventajas}

El modelo consta de 6 slots a diferencia del anterior de solo 3 .

Elimina el tener que agacharse para llenar el molde con arcilla.

Se adapta a cualquier persona respecto a la altura.
Cuenta con agarraderas y llantas, permitiendo transportar el molde a cualquier parte.

A diferencia del molde anterior en este no es necesario ajustar la mezcla en cada slot, en este modelo al momento de cerrar la cubierta que contienen la ranura del ladrillo, automáticamente saca el excedente y alinea los ladrillos.

\section{Desventajas}

Es una sola pieza.

Para la que su uso sea correcto y práctico se deberán utilizar plásticos que servirán como base de los ladrillos y poder transportarlos por la rampa que tiene el molde.

\section{Recomendaciones}

En el diseño de la ranura, se recomienda dejarla rectangular y no de una forma personalizada ya que, con esto, aparte de que se reduce más el material utilizado para el ladrillo, también facilita la elaboración de los ladrillos.

El diseño está basado principalmente en madera, se recomienda en un futuro fabricar el molde de acero inoxidable ya que esto le dará una mayor durabilidad al molde.

Analizar la composición de los elementos para la fabricación de los ladrillos como es Arcilla, agua y aserrín.

Verificar la temperatura de cocimiento de los ladrillos y su método.

\section{Conclusión}

El éxito de la utilización de la metodología DMAIC depende no sólo de los conocimientos en métodos estadísticos sino del compromiso de los dueños para poder liderar este cambio. $\mathrm{La}$ situación que se encontró en este tipo de negocios, es que no se cumplía con los requisitos necesarios ergonómicos y de infraestructura para poder elevar la productividad, además no contaba con una gestión de calidad efectiva. 
Se pretende que la productividad aumente de un $40 \%$ a un $80 \%$ y que el trabajador no se lesione por el uso de moldes anti ergonómicos y tenga una mejor calidad de vida.

\section{Referencias}

Barranzuela, E (2014). Proceso productivo de los ladrillos de arcilla producidos en la Región Piura.

Campbell, J(2004). Ladrillo historia universal, Barcelona, 2004.

Denue, 2018 (Directorio Estadístico Nacional de unidades económicas). Recuperado de: https://www.inegi.org.mx/app/mapa/denue/

Donderis, L(2019).Application of the first three stages of the DMAIC methodology to identify the main cause of decline in the production process of wheat flour tortillas.

González, F (2003). Seis Sigma. Editorial Libros en Red.

Gutiérrez, H (2009). Control Estadístico de Calidad y Seis Sigma. Editorial. Mc Graw Hill. Inegi (Censos Económicos 2013). Retrieved from Instituto Nacional de Estadística y Geografía:

http://www.inegi.org.mx/est/contenidos/espanol /proyectos/censos/ce2009/p

rivadoparaestatal.asp

Inegi, 2018. Scince (Sistema para la consulta de información censal).

López, J(2015). Gestión y Arranque de línea de producción. Aguascalientes.

Melo, J(2009). Ergonomía Practica. Guía para la evaluación ergonómica de un puesto de trabajo. Buenos Aires Argentina.

Navarro, G (2018). Estudio Socioeconómico del Estado de Chihuahua y de Cd. Juárez para la Especialidad en Sistemas Energéticos Optimizados (IELE-SEO-2016-01) de la Carrera de Ingeniería Eléctrica (IELE-2010-209) en el ITCJ.

Norma Mexicana "NMX-C-404-ONNCCE2012". Bloques, tabiques o ladrillo tabicones para uso estructural - especificaciones y métodos de ensayo
Norma Mexicana "NMX-C-036-ONNCCE2013". Resistencia a la compresión de bloque de tabique o ladrillos y tabicones y adoquinesmétodo de ensayo

Norma Mexicana "NMX-C-006-1976, Ladrillos y Bloques Cerámicos de Barro, Arcilla y/o Similares.

Norma Mexicana" NMX-C-404-1997ONNCCE, Industria de la ConstrucciónBloques, Tabiques o ladrillos y Tabicones para Uso Estructural-Especificación y Métodos de Prueba.

Pulido, H(2008). Control estadístico de calidad y seis sigma. Editorial McGraw-Hill.

Semarnat, INECC(2016). Análisis de mercado del sector de construcción y proyecto Piloto a nivel Región.

Sánchez, M(2013), El origen de los ladrillos Recuperado de: https://ladrillos.es/el-origen-delos-ladrillos/

Servicios Profesionales para el Desarrollo Económico, S.C SERpro (2012). Diagnostico Nacional del sector ladrillero artesanal de México. Recuperado de: http://www.redladrilleras.net/assets/files/692eca a0a857372af35a529441387778.pdf

Scince, 2010. Sistema para la consulta de información Censal. Recuperado de: http://gaia.inegi.org.mx/scince2/viewer.html. 